\title{
Pyrazine-chromene-3-carbohydrazide conjugates: Molecular docking and ADMET predictions on dual- acting compounds against SARS-CoV-2 Mpro and RdRp
}

\author{
Arif MERMER $1 *$ (D), Serhii VAKAL 2 (D) \\ 1 Experimental Medicine Research and Application Center, University of Health Sciences-Turkey, 34660, Turkey \\ 2 Structural Bioinformatics Laboratory, Biochemistry, Faculty of Science and Engineering, Åbo Akademi University, \\ Tykistökatu 6A, FI-20520, Turku, Finland. \\ * Corresponding Author. E-mail: arif.mermer@sbu.edu.tr (A.M.); Tel. +90-216-418 9616.
}

Received: 28 April 2021 / Revised: 15 November 2021 / Accepted: 18 November 2021

\begin{abstract}
Drug discovery campaigns against COVID-19 lag far behind vaccine development, but given the low vaccine production rate and unfair distribution, there is still an urgent need to advance reliable and potent anti-SARSCoV-2 agents. We aimed to identify novel and effective molecules with dual-target activity against SARS-CoV-2 main protease (Mrro) and RNA-dependent RNA-polymerase (RdRp). For this, we designed and evaluated the library of hybrid compounds based on pyrazine and $4 \mathrm{H}$-chromen-4-one linked by amide bridges. The synthetic availability of the compounds ranged from 3.08 to 3.40, indicating that these compounds are easy to synthesize. According to in silico ADMET prediction, most of the compounds satisfied all rules of drug-likeness. Compounds CPC-2, 3, 4, 8, 10, 11-14, and 16 were CYP1A2, CYP2C9, and CYP3A4 inhibitors, whereas none of them inhibited CYP2C19 and CYP2D6 isoforms. All designed compounds were predicted to be well-absorbed in the GI tract but not blood-brain barrier permeant and not subject to active efflux.

Molecular docking studies against SARS-CoV-2 Mpro showed that compounds CPC-1, 6, 7, 8, and 10 could establish multiple H-bonds with the binding site residues. In the case of SARS-CoV-2 RdRp, compounds CPC-5, 6, 8, 13, 14, and 16 had the most favorable binding orientations and could establish $\mathrm{H}$-bonds, pi-cation, and salt-bridges with the binding tunnel residues and RNA. Compound CPC- 6 turned to be the most promising candidate from the dual-action side since it had reasonable docking scores and MM-GBSA $\Delta \mathrm{G}_{\text {bind }}$ values, and good interaction profiles for both Mpro and RdRp.
\end{abstract}

KEYWORDS: Pyrazine; chromen-4-one; ADMET; molecular docking; SARS-CoV-2 Mpro; SARS-CoV-2 RdRp.

\section{INTRODUCTION}

Coronaviruses are spherical positive RNA viruses in the Coronaviridae family, with the largest genome among all RNA viruses [1]. Coronavirus (SARS-CoV), which emerged in 2003, remained an epidemic, while the novel Coronavirus (SARS-CoV-2), which emerged in Wuhan, China, in November 2019, has rapidly spread all over the world and become a pandemic [2]. As of 27 April 2021, there have been 147.377.159 confirmed infected Covid-19 cases, containing 3.112.041 directly or related deaths, reported to WHO (who.sprinklr.com/), and, unfortunately, the number of cases continues to increase day by day, as well. Many vaccine and drug studies are ongoing worldwide to overcome this pandemic, but promising results have been obtained in vaccine studies only, while drug development campaigns have not yet reached a conclusion. However, reasons such as the slow vaccination rate, the opposition to vaccines by society, and the unfair distribution of vaccines worldwide (developed countries have easier access than underdeveloped countries) point out that the pandemic will last longer than expected. Thus, there is still an urgent need to advance reliable and potent anti-SARS-CoV-2 agents.

The enzyme 3-chymotrypsin-like protease (3CLpro), also known as the main protease enzyme (Mpro), cleaves at least 11 sites on the polyproteins translated from the viral RNA of SARS-CoV-2, while it also hydrolyses the viral polyproteins pp1a and pp1ab to generate functional proteins during coronavirus replication. Mpro has been validated as a potential target for enhancing drugs to treat SARS-CoV-2 due to its highly preserved sequence and primary functional features [3-6]. RNA-dependant RNA-polymerase (RdRp),

How to cite this article: Mermer A, Vakal S. Pyrazine-chromene-3-carbohydrazide conjugates: Molecular docking and ADMET predictions on dual-acting compounds against SARS-CoV-2 Mpro and RdRp. J Res Pharm. 2021; 25(6): 953-966. 
being the essential component of the replication \& transcription machinery, is a crucial enzyme for all RNA viruses, including coronaviruses [7]. Due to this, $\mathrm{RdRp}$ is an attractive therapeutic target, and a few inhibitors of RdRp have been designed recently [8].

Many studies are in progress, using various approaches to determine potent therapeutics against this virus. For example, chloroquine, an immunomodulant drug used in the treatment of malaria, has been predicted to be effective in reducing viral replications against coronavirus in in vitro studies $[9,10]$. Many countries have utilized hydroxychloroquine, an analog of chloroquine, at the beginning of the pandemic [11] (Figure 1). Another drug, Favipiravir, a carboxamide derivative that functions to selectively inhibit the RNAdependent RNA-polymerase of the influenza virus [12], is used to reduce hospitalizations of infected patients and to overcome the disease more quickly. However, in recent studies, hydroxychloroquine is not suggested because of the worst side effects such as gastrointestinal tracts symptoms. Although favipiravir was used by many countries in the treatment at the beginning of the pandemic, its use has decreased with the development of vaccines. Further, there are many other already-known and novel drugs in clinical trials against SARS-CoV2, such as Indinavir, Saquinavir, Darunavir, ASC09, Ritonavir, Lopinavir, Remdesivir, and Ivermectine [13].<smiles>CCN(CCO)CCCC(C)Nc1ccnc2cc(Cl)ccc12</smiles>

Hydroxychloroquine

Darunavir<smiles>CCN(CC)CCCC(C)Nc1ccnc2cc(Cl)ccc12</smiles><smiles>NC(=O)c1nc(F)cnc1O</smiles>

Figure 1. Some of the earlier-known drugs that have been used against SARS-CoV-2.

In drug discovery studies, many parameters such as which reactants to use and in which amount, substituent selection, and whether they are biologically active or not are evaluated to avoid time and resource waste. The molecule to be synthesized must have high activity and low toxicity at the same time. The conventional step to consider pharmacokinetics is to separate the diverse impacts affecting the access to the target into individual parameters. In this context, these ADMET (for Absorption, Distribution, Metabolism, Excretion, and Toxicity) parameters can be evaluated separately by reliable methods. In drug design and discovery studies, it has been shown that the early estimation of ADMET properties dramatically reduces the potential disadvantages in clinical stages. Computer models have been encouraged as an effective alternative to experimental procedures to estimate ADMET, particularly at early steps, when studied chemical structures are countless, but the availability is limited $[14,15]$.

The SwissADME is a web-based platform that is freely accessible at http://www.swissadme.ch and can be used even by non-experts in computer-aided drug design (CADD) studies, and the results can be easily analyzed. Compared to other web-based tools for determining ADME and pharmacokinetic properties, SwissADME key points, in particular, are: various input options, computation for multiple compounds, and the opportunity to show, save and share outcomes per individual compounds or through global intuitive and interactive graphs. Consequently, SwissADME is combined with the SwissDrugDesign suite. One-click interoperability gains access to different CADD tools improved by the Molecular Modeling Group of the SIB Swiss Institute of Bioinformatics, e.g., ligand-based virtual screening, biotarget prediction, molecular docking, bioisosteric design, or molecular mechanics [16-24].

Docking-based virtual screening is a standard strategy used in molecular discovery to screen large chemical libraries and identify molecular structures that are most likely to bind to a specific biological target [23]. Up to date, more than a hundred papers involving the docking-based discovery of SARS-CoV-2 Mpro or 
RdRp have been published (to name a few best cited - [7,26-28]). The recent concept of multi-target drug design provides some attractive benefits, such as higher resistance tolerance, lower application dose, and toxicity [29]. To the best of our knowledge, there was only one study dealing with discovering the dualinhibitors of SARS-CoV-2 enzymes [30].

In this study, we aimed to identify novel and effective molecules for SARS-CoV-2 infection treatment. For this, we designed and evaluated the library of hybrid compounds based on pyrazine and $4 \mathrm{H}$-chromen-4one. We primarily looked at the structures of drugs currently used to treat COVID-19 and some other antiviral drugs (Figure 2). We hypothesized that the designed compound, including pyrazine ring and chlorine as a substituent in the presently used drugs such as favipiravir, chloroquine, and hydroxychloroquine, and the coumarin analogs found in many antiviral agents can potentially show dual-target activity and would be worthy of exploring [31,32]. Furthermore, the amide bridges as linkers in many compounds play an essential role in biological activity by establishing hydrogen bonds with the active site of the target proteins [33]. Therefore, we used favipiravir and $4 H$-chromen-4-one core and amide bridges as a linker to study against SARS-CoV-2 Mpro and RdRp in silico.
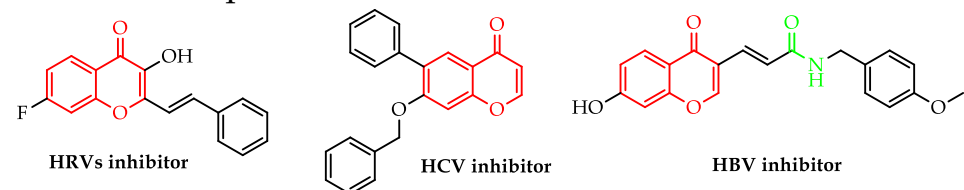

$$
\text { Favipavir }
$$
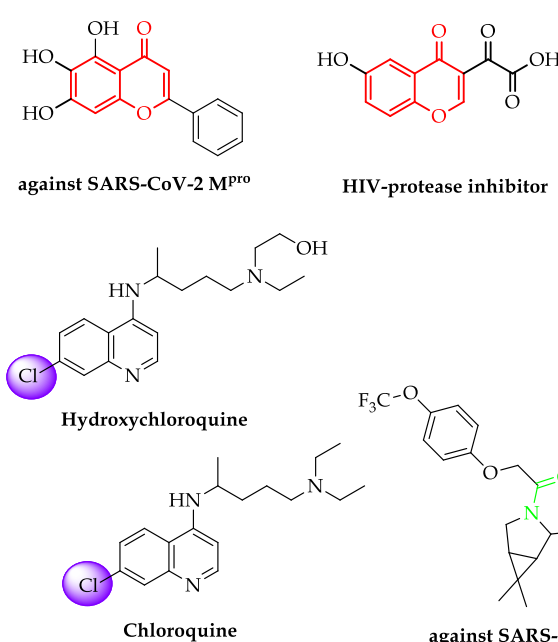

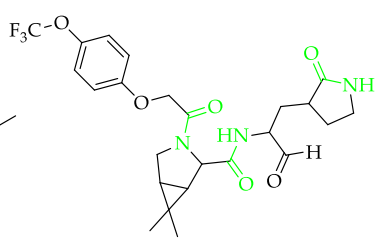

against SARS-CoV-2 $\mathrm{M}^{\text {pro }}$
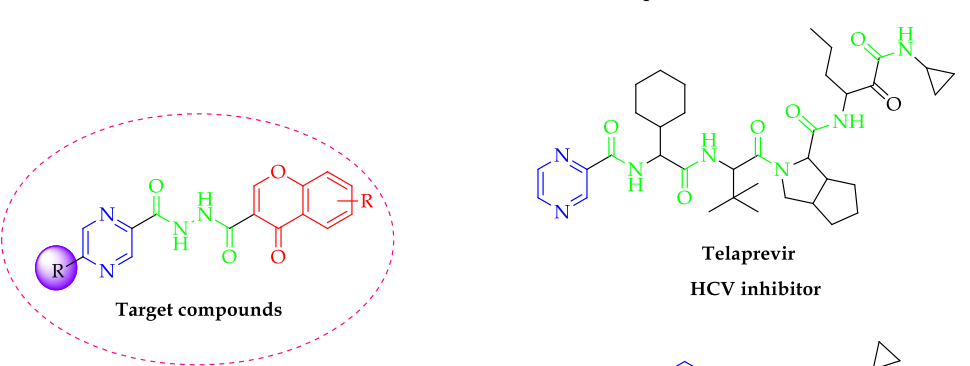

Figure 2. Pyrazine, 4H-chromen-4-one and amide bridge containing antiviral agents and target compound structure.

\section{RESULTS and DISCUSSION}

\subsection{In silico ADME Prediction}

The ADME prediction for the 16 novel designed compounds was performed with the SwissADME tool, and a set of physicochemical properties, including hydrogen-bond acceptors $(n \mathrm{HA})$, hydrogen-bond donors $(n \mathrm{HD})$, and topological polar surface area (TPSA), were calculated. Besides, lipophilicity (iLOGP, XLOGP3, WLOGP, MLOGP, SILICOS-IT, and Consensus $P_{\mathrm{o}}$ w), water-solubility properties (ESOL, Ali, SILICOS-IT), synthetic accessibility, and drug-likeness factors (Lipinski's Rules, Ghose, Veber, Egan, Muegge) were also predicted. All designed compounds showed high topological polar surface area (TPSA) ranging from 114.19 $\AA^{2}$ to $160.01 \AA^{2}$, and these compounds were found to be highly soluble or at least moderately soluble with the Log $S$ values ranging from -2.62 to $-4.25 \mathrm{mg} / \mathrm{mL}$. The number of $\mathrm{HD}(n \mathrm{HD})$ of the compounds was between 2 and 4 , whereas the $n \mathrm{HA}$ was between 6 and 9 . The presence of $\mathrm{HD}$ and HA groups in a compound may suggest that this compound can establish H-bonds with the target protein's active site. Accordingly, the molecular docking results demonstrated that the designed compounds make $\mathrm{H}$-bonds with specific amino acids in the SARS-CoV-2 Mpro enzyme. The calculated lipophilicity properties were given in a consensus model (n-octanol and water: $\log P_{\mathrm{o} / \mathrm{w}}$ ), which was ranging from 0.27 to 2.06. Pharmacokinetic properties such as GI absorption, BBB permeability, P-gp substrate-likeness, and skin permeation ( $\log \mathrm{Kp}$ ) were also calculated, and the designed compounds exhibited high Gastrointestinal absorption (GI) except for compounds CPC-6, CPC-7, 
CPC-14, and CPC-15 which include nitro or dihydroxy as a substituent in the chromene core, while none of them showed blood-brain barrier permeability. Skin permeation kinetics (Log Kp) were from -6.82 to -8.02 $\mathrm{cm} / \mathrm{s}$, as shown in Table 1. Moreover, the synthetic availability of the designed compounds ranges from 3.08 to 3.40, indicating that these compounds are easy to synthesize (values range from 1 (very easy) to 10 (very difficult)).

Table 1. Predicted physiochemical properties of the designed compounds.

\begin{tabular}{|c|c|c|c|c|c|c|c|c|}
\hline $\begin{array}{l}\text { Comp. } \\
\text { No }\end{array}$ & TPSA $\left(\AA^{2}\right)$ & $n \mathrm{HD}$ & $n \mathbf{H A}$ & $\log P_{\mathrm{o} / \mathrm{w}}$ & $\log S$ & $\begin{array}{c}\log K p \\
(\mathrm{~cm} / \mathrm{s})\end{array}$ & PAINS & $\begin{array}{c}\text { Synthetic } \\
\text { accessibility }\end{array}$ \\
\hline CPC-1 & 134.42 & 3 & 8 & 0.67 & -2.76 & -7.68 & 0 & 3.12 \\
\hline СРC-2 & 114.19 & 2 & 7 & 1.52 & -3.20 & -7.15 & 0 & 3.25 \\
\hline CPC-3 & 114.19 & 2 & 7 & 1.63 & -3.81 & -7.31 & 0 & 3.18 \\
\hline СРС-4 & 114.19 & 2 & 7 & 1.75 & -3.50 & -7.09 & 0 & 3.11 \\
\hline CPC-5 & 123.42 & 2 & 8 & 0.99 & -2.97 & -7.53 & 0 & 3.21 \\
\hline СРC-6 & 154.65 & 4 & 9 & 0.27 & -2.62 & -8.02 & 1 & 3.18 \\
\hline СРC-7 & 160.01 & 2 & 9 & 0.32 & -2.96 & -7.72 & 0 & 3.23 \\
\hline СРC-8 & 132.65 & 2 & 9 & 1.21 & -3.04 & -7.73 & 0 & 3.40 \\
\hline СРС-9 & 134.42 & 3 & 7 & 0.91 & -3.20 & -7.40 & 0 & 3.08 \\
\hline CPC-10 & 134.42 & 2 & 6 & 1.73 & -3.64 & -6.88 & 0 & 3.21 \\
\hline CPC-11 & 114.19 & 2 & 6 & 2.06 & -4.25 & -7.04 & 0 & 3.12 \\
\hline CPC-12 & 114.19 & 2 & 6 & 1.87 & -3.93 & -6.82 & 0 & 3.08 \\
\hline CPC-13 & 123.42 & 2 & 7 & 1.36 & -3.41 & -7.25 & 0 & 3.17 \\
\hline CPC-14 & 154.65 & 4 & 8 & 0.53 & -3.05 & -7.75 & 1 & 3.14 \\
\hline CPC-15 & 160.01 & 2 & 8 & 0.55 & -3.39 & -7.45 & 0 & 3.19 \\
\hline CPC-16 & 132.65 & 2 & 8 & 1.42 & -3.48 & -7.46 & 0 & 3.36 \\
\hline
\end{tabular}

Another essential piece of information is the possibility of interaction with cytochromes P450 (CYP). This superfamily of isoenzymes is a crucial player in drug elimination through metabolic biotransformation [34]. It has been proposed that CYP and P-gp can synergistically commit small compounds to prevent their action in tissues and organisms [35]. One can anticipate that 50 to $90 \%$ (depending on the authors) of medicinal compounds are substrates of five substantial CYP isoforms (CYP1A2, CYP2C19, CYP2C9, CYP2D6, CYP3A4) [36,37]. Considering our results, the compounds CPC-2, 3, 4, 8, 10, 11-14 and 16, which contain a halogen, methyl, or methoxy group as a substituent in chromene and chlorine or fluorine linked to pyrazine core, were found to be CYP1A2, CYP2C9 and CYP3A4 inhibitors, whereas none of the designed compounds exhibited positive results for CYP2C19 and CYP2D6 isoforms (Table 2).

Drug-likeness defines qualitatively the capability of a compound to be an oral drug in accordance with bioavailability. Drug-likeness was determined from structural or physicochemical controls of improvement compounds developed to be oral drug candidates. Swiss-ADME supplies five different rule-based filters: Lipinski, Ghose, Veber, Egan, and Muegge. All of the designed compounds obey the Lipinski and Ghose rules. On the other hand, compounds CPC-1, 6, 7, 14, and 15 violated the Veber, Egan, and Muegge rules due to high TPSA results, which have to be $\leq 140, \leq 131.6$, and $\leq 150$, respectively. Furthermore, the bioavailability radar of the compounds is given in Figure 3, and the colored zone depicts suitable physicochemical space for oral bioavailability.

Table 2. Predicted pharmacokinetics and drug-likeness of the designed compounds.

\begin{tabular}{lllllllllllllllll}
\hline Compounds (CPC) & $\mathbf{1}$ & $\mathbf{2}$ & $\mathbf{3}$ & $\mathbf{4}$ & $\mathbf{5}$ & $\mathbf{6}$ & $\mathbf{7}$ & $\mathbf{8}$ & $\mathbf{9}$ & $\mathbf{1 0}$ & $\mathbf{1 1}$ & $\mathbf{1 2}$ & $\mathbf{1 3}$ & $\mathbf{1 4}$ & $\mathbf{1 5}$ & $\mathbf{1 6}$ \\
\hline Pharmacokinetics & & & & & & & & & & & & & & & \\
\hline GI absorption & $\uparrow$ & $\uparrow$ & $\uparrow$ & $\uparrow$ & $\uparrow$ & $\downarrow$ & $\downarrow$ & $\uparrow$ & $\uparrow$ & $\uparrow$ & $\uparrow$ & $\uparrow$ & $\uparrow$ & $\downarrow$ & $\downarrow$ & $\uparrow$ \\
BBB permeability & - & - & - & - & - & - & - & - & - & - & - & - & - & - & - & - \\
CYP1A2 inhibitor & - & + & + & + & - & - & - & + & - & + & + & + & + & - & - & + \\
CYP2C19 inhibitor & - & - & - & - & - & - & - & - & - & - & - & - & - & - & - & - \\
CYP2C9 inhibitor & - & + & + & + & - & - & - & + & - & + & + & + & + & - & + & + \\
CYP2D6 inhibitor & - & - & - & - & - & - & - & - & - & - & - & - & - & - & - & - \\
CYP3A4 inhibitor & - & + & + & + & + & - & - & + & - & + & + & + & + & - & - & + \\
Lipinski & + & + & + & + & + & + & + & + & + & + & + & + & + & + & + & + \\
Ghose & + & + & + & + & + & + & + & + & + & + & + & + & + & + & + & + \\
Veber & + & + & + & + & + & - & - & + & + & + & + & + & + & - & - & + \\
Egan & - & + & + & + & + & - & - & - & - & + & + & + & + & - & - & - \\
Muegge & + & + & + & + & + & - & - & + & + & + & + & + & + & - & - & + \\
\hline
\end{tabular}



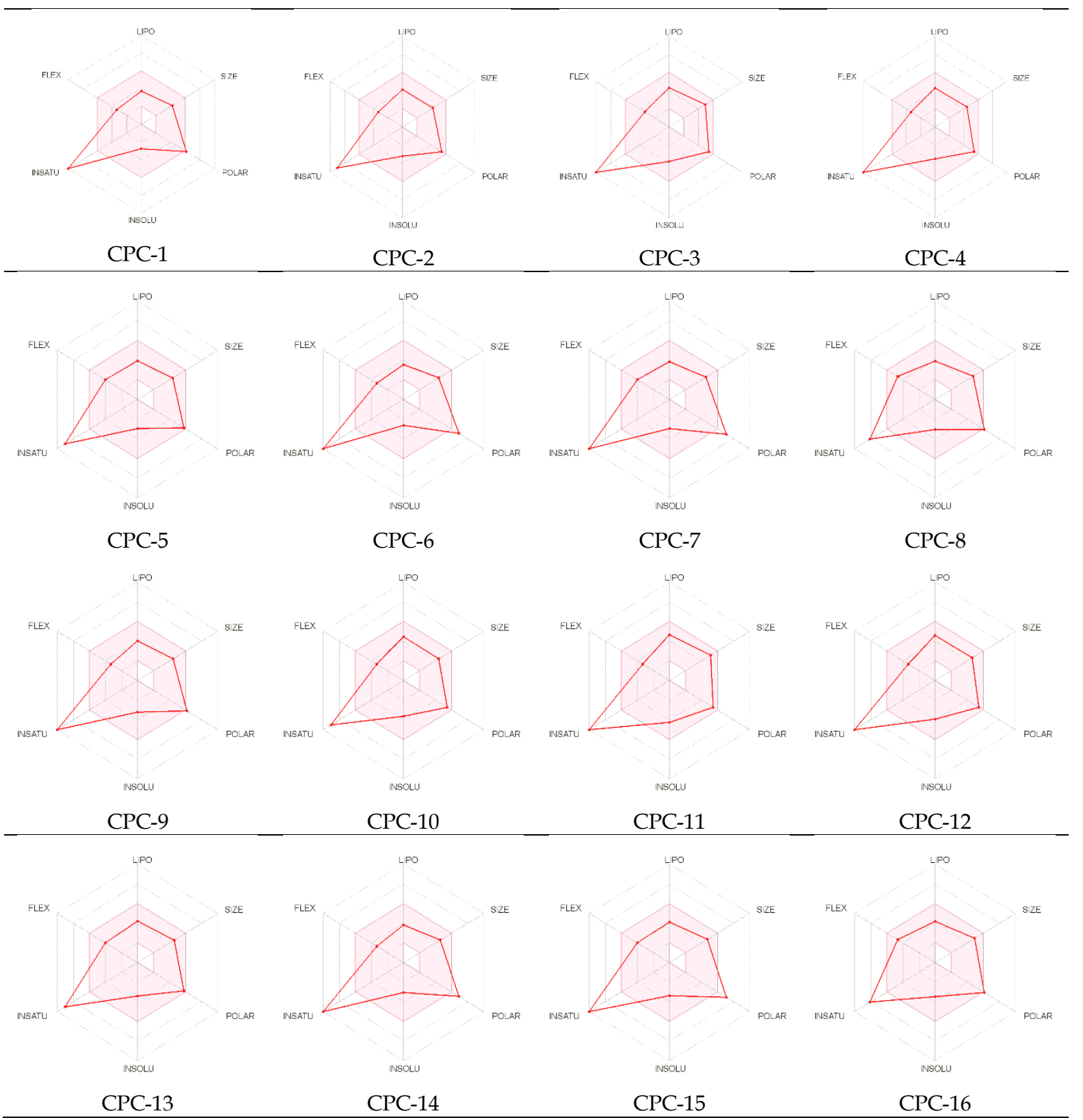

Figure 3. The Bioavailability Radar gives a first glance at the drug-likeness of the compounds. The pink area represents the optimal range for each property (lipophilicity: XLOGP3 between -0.7 and +5.0 , size: MW between 150 and $500 \mathrm{~g} / \mathrm{mol}$, polarity: TPSA between 20 and $130 \AA^{2}$, solubility: $\log S$ not higher than 6 , saturation: fraction of carbons in the $\mathrm{sp}^{3}$ hybridization not less than 0.25 , and flexibility: no more than 9 rotatable bonds.

Furthermore, the BOILED-Egg profile, which enables the intuitive consideration of passive gastrointestinal absorption (HIA) and brain penetration (BBB) in the function of the position of the molecules in the WLOGP-vs.-TPSA referential was screened for the selected five compounds [38]. The white area corresponds to a high probability of passive absorption in the GIT, while the yellow area is for a high probability of brain penetration. Also, the marks are colored in blue if predicted as actively effluxed by P-gp (PGP+) and in red if estimated as non-substrate of P-gp (PGP-). All designed compounds were estimated to be well-absorbed but not accessing the brain, and all compounds, except for CPC-8 that includes a dimethoxy group in chromene core and fluorine in pyrazine and is subjected to active efflux (blue dot), were not subject to active efflux (red dot) (Figure 4). 


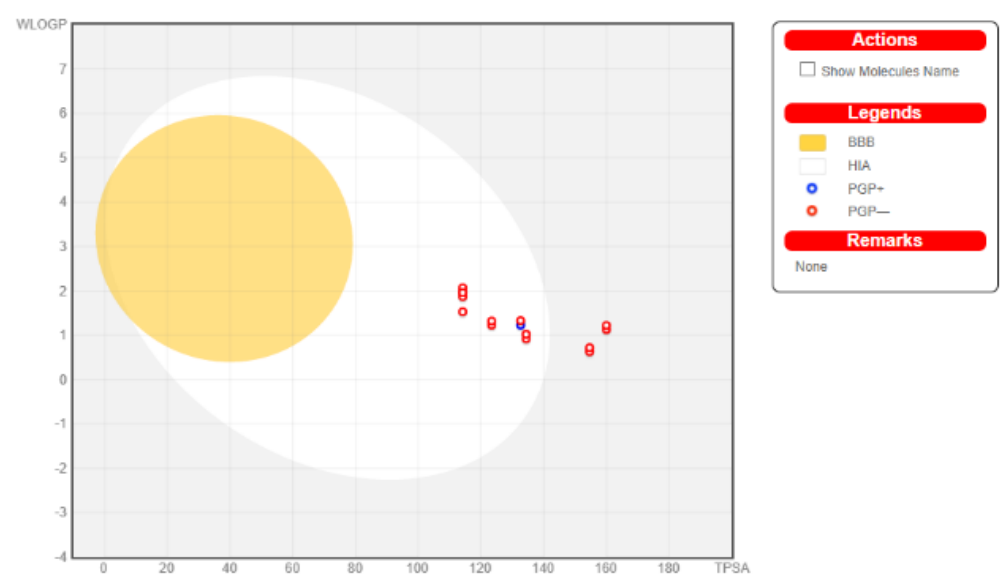

Figure 4. BOILED-Egg presentation of the compounds.

\subsection{Target Prediction}

The target estimation of all compounds was performed using the SwissTargetPrediction platform with the following investigations of the results depicted as a pie-chart (Figure 5). The CPC-1 and CPC-9, containing respectively hydroxy and fluorine and hydroxy and chlorine linked to chromene and pyrazine structure as a substituent, were predicted as 33\%, $40 \%$ of protease. In contrast, CPC-2 with methyl in chromene ring and fluorine in pyrazine was predicted as $40 \%$ of Family A G-protein-coupled receptor. CPC-6 and CPC-15 were estimated as lyases with 27\%; also, compound CPC-14 was found to be $20 \%$ of phosphatase. All other compounds were predicted as kinase inhibitors with varying percentages, as given in Figure 5. One of the compound's (CPC-10) result table comprising Common Name, Uniprot ID, Target, ChEMBL-ID, Probability, Target Class, and Known actives in 2D/3D are given in the Supporting Information file (Figure S1).

\subsection{Toxicity Prediction}

AMES test is a widely utilized process to determine a compound mutagenic potential using bacteria. A positive test demonstrates that the compound is mutagenic and thus may behave as a carcinogen. The maximum recommended tolerated dose (MRTD) serves as a prediction of the toxic dose threshold of chemicals in humans, and for a better MRTD has to be $\leq 0.477 \log (\mathrm{mg} / \mathrm{kg} /$ day). Skin sensitization is a probable side effect for a dermal application of a natural or synthetic compound. T. Pyriformis is a protozoan with its toxicity generally used as a toxic measure, and if the results are higher than $-0.5 \log \mu \mathrm{g} / \mathrm{L}$, a compound can be considered toxic. Furthermore, if Minnow $\mathrm{LD}_{50}<-0.3$, it can be regarded as high acute toxicity (http:// biosig.unimelb.edu.au/pkcsm/). Hepatoxicity represents drug-induced liver injury. According to our toxicity predictions, compounds CPC-7, CPC-11, and CPC-15 showed negative AMES toxicity. Compounds CPC-1, 5, 6, 8, 9, 13, 14, and 16 violated the MRTD, while other compounds were found to be suitable. None of the compounds was predicted to be a hERG inhibitor. The designed compounds showed acute and chronic oral rat toxicity with varying $\mathrm{LD}_{50}$ and LOAEL results. The compounds exhibited hepatoxicity, whereas they were not predicted to cause skin sensitization (Table 3).

\subsection{Molecular Docking}

At first, validation re-docking was performed using different protocols and a complex of SARS-CoV-2 Mpro with inhibitor telaprevir (PDB id - 6ZRT). Glide XP without explicit constraints could successfully reproduce the crystallographic binding orientation of telaprevir with RMSD $<1.0 \AA$ with a correct dominant cluster of poses, so it was selected as the main protocol for the docking of studied compounds. 10 out of 16 compounds could be docked into the orthosteric site of Mpro, namely, CPC-1, 3, 4, 6, 7, 8, 10, 12, 14, and 15. The best docking score was achieved by compounds CPC-6, 14, 1, and 8: -7.17, -6.61, -6.45, and -6.06, respectively (XP g-score of a reference ligand was -8.26). However, CPC-14 could not establish enough H-bonds with the 
active site residues, so it was excluded from further analysis. Most of the compounds established H-bond between the secondary amine group and $\mathrm{H} 164$ residue (Figure 6). CPC-6 had the best binding orientation since it established four H-bonds throughout the whole extended molecule, and such binding mode should have made the molecule well-fixed in the Mpro active site: pyrazine ringed H-bonded with G140, secondary amine group interacted with H164, 6,7-dihydroxychromen-4-one had two H-bonds with T190, and three keto-groups were exposed to the solvent. CPC-1 and CPC-7 also seem to have rigid poses, and each of them establishes three H-bonds with G140, H164, and T190 (CPC-1) or H164, E166, and Q192 (CPC-7). MM-GBSA calculations showed $\Delta \mathrm{G}_{\text {bind }}$ values in the range from -67.3 (best; CPC-15) to -45.4 (worst; CPC-14) kcal/mol. The compounds with favorable binding poses (Figure 6) also had high $\Delta \mathrm{G}_{\text {bind }}$ values: -55.8 (CPC-1), -53.3 (CPC-6), -63.8 (CPC7), -59.4 (CPC-8), and -59.4 (CPC-10) $\mathrm{kcal} / \mathrm{mol}$. Such highly negative values indicate that the selected compounds can potentially bind strongly to the SARS-CoV-2 Mpro active site (Table S1).

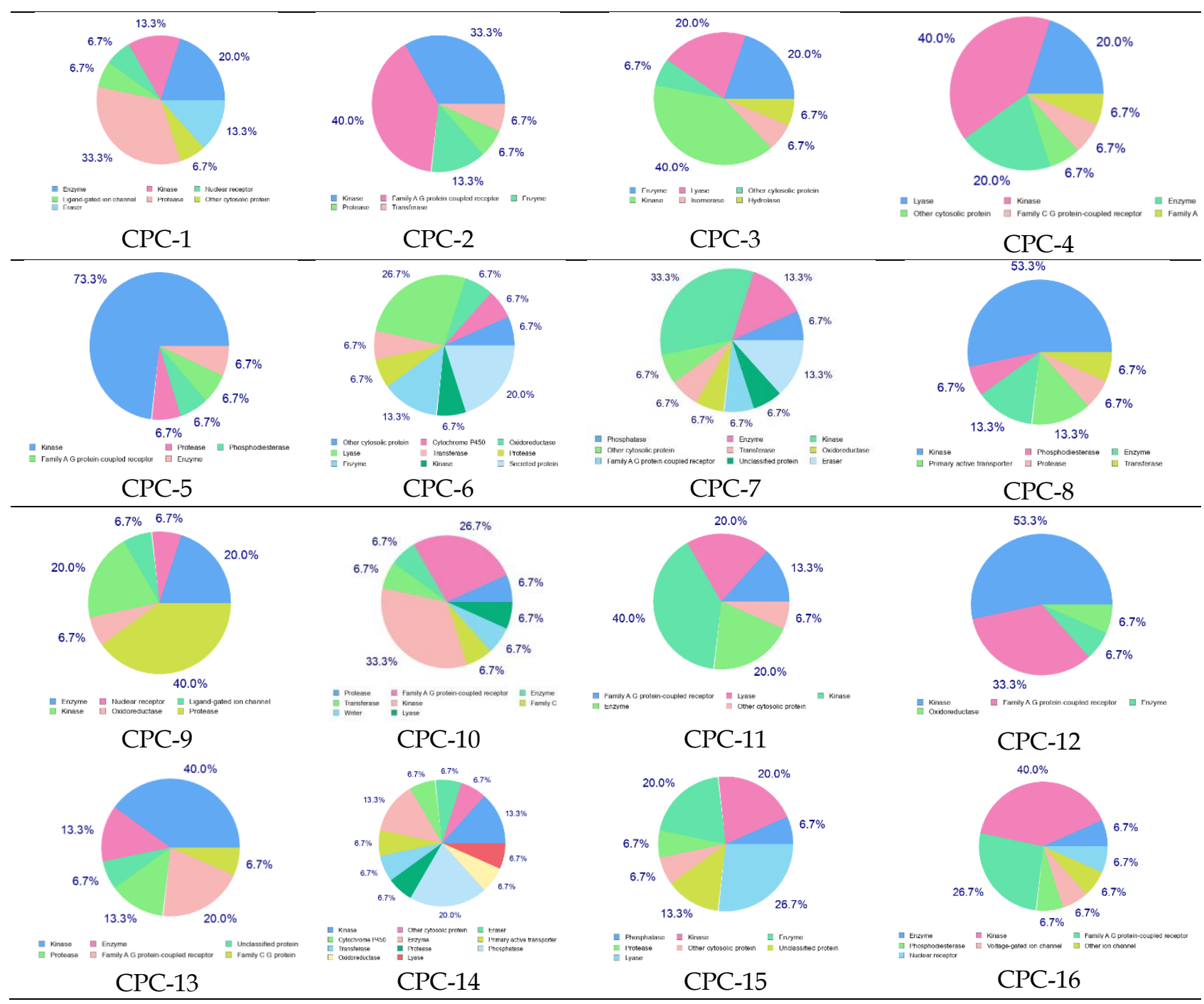

Figure 5. SwissTargetPrediction of the designed compounds.

Table 3. Toxicity prediction results for the selected compounds. 


\begin{tabular}{|c|c|c|c|c|c|c|c|c|c|c|}
\hline Comp. & $\begin{array}{l}\text { AMES } \\
\text { tox. }\end{array}$ & $\begin{array}{l}\text { Max. } \\
\text { Tolerated } \\
\text { Dose }\end{array}$ & $\begin{array}{l}\text { hEGR } \\
\text { I inh. }\end{array}$ & $\begin{array}{l}\text { hEGR } \\
\text { II inh. }\end{array}$ & $\begin{array}{l}\text { Oral Rat } \\
\text { Acute } \\
\text { Tox. } \\
\left(L_{50}\right)\end{array}$ & $\begin{array}{l}\text { Chronic } \\
\text { Oral Rat } \\
\text { Tox. } \\
\text { (LOAEL) }\end{array}$ & Hepatox. & $\begin{array}{l}\text { Skin } \\
\text { Sens. }\end{array}$ & $\begin{array}{l}\text { T.Pyriformis } \\
\text { tox. }\end{array}$ & $\begin{array}{l}\text { Minnow } \\
\text { tox. }\end{array}$ \\
\hline CPC-1 & $\mathrm{No}$ & 0.896 & No & $\mathrm{No}$ & 1.748 & 1.656 & Yes & No & 0.295 & 2.006 \\
\hline CPC-2 & No & 0.465 & No & No & 1.638 & 1.685 & Yes & No & 0.309 & 1.172 \\
\hline CPC-3 & No & 0.465 & No & No & 1.651 & 1.635 & Yes & No & 0.306 & 0.808 \\
\hline CPC-4 & No & 0.473 & No & No & 1.641 & 1.641 & Yes & No & 0.306 & 0.954 \\
\hline CPC-5 & No & 0.659 & No & No & 1.623 & 1.693 & Yes & No & 0.292 & 1.434 \\
\hline CPC-6 & No & 1.072 & No & No & 1.973 & 2.041 & Yes & No & 0.286 & 2.114 \\
\hline CРC-7 & Yes & 0.367 & No & No & 2.016 & 1.746 & Yes & No & 0.292 & 0.479 \\
\hline CPC-8 & No & 0.791 & No & No & 1.662 & 2.013 & Yes & No & 0.286 & 1.671 \\
\hline СРС-9 & No & 0.869 & No & No & 1.791 & 1.620 & Yes & No & 0.296 & 1.673 \\
\hline CPC-10 & No & 0.428 & No & No & 1.694 & 1.649 & Yes & No & 0.311 & 0.839 \\
\hline CPC-11 & Yes & 0.429 & No & No & 1.704 & 1.599 & Yes & No & 0.309 & 0.475 \\
\hline CPC_12 & No & 0.437 & No & No & 1.694 & 1.606 & Yes & No & 0.309 & 0.621 \\
\hline CPC-13 & No & 0.622 & No & No & 1.673 & 1.658 & Yes & No & 0.294 & 1.101 \\
\hline CPC-14 & No & 1.505 & No & No & 1.996 & 2.005 & Yes & No & 0.286 & 1.781 \\
\hline СРC-15 & Yes & 0.351 & No & No & 2.048 & 1.711 & Yes & No & 0.293 & 0.145 \\
\hline CPC-16 & No & 0.754 & No & No & 1.704 & 1.978 & Yes & No & 0.286 & 1.337 \\
\hline
\end{tabular}

a

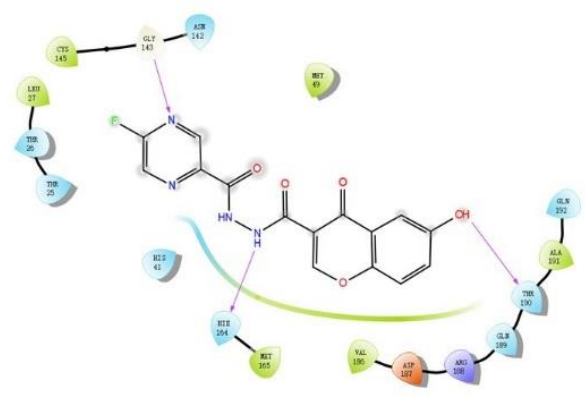

C

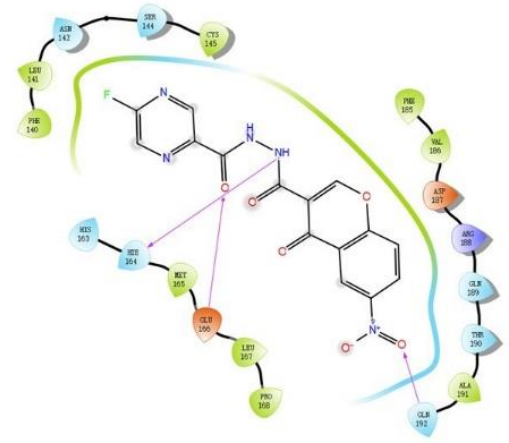

b

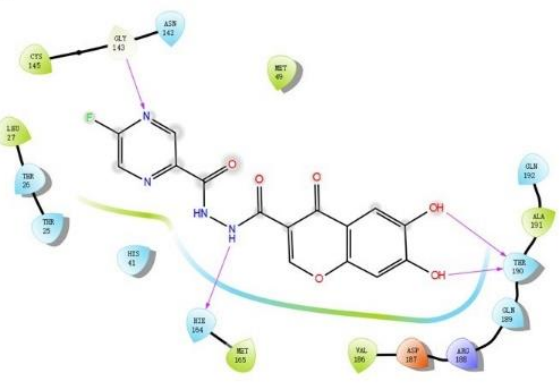

d

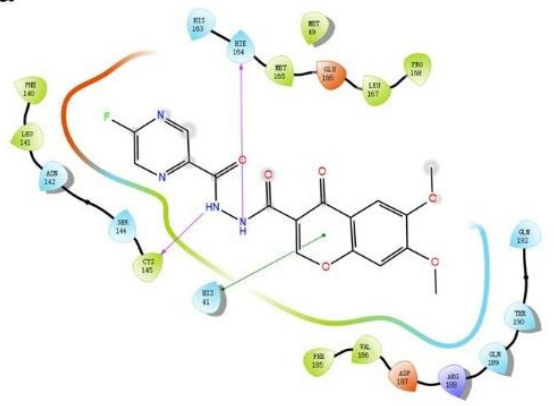

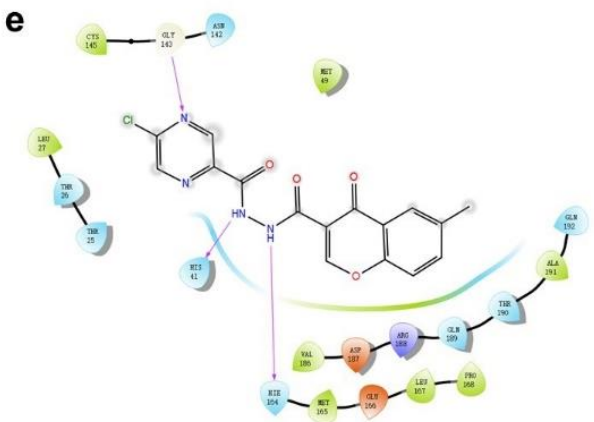

Figure 6. Interaction diagrams for the compounds with the highest predicted affinity to SARS-CoV-2 Mpro. a) CPC-1, b) CPC-6, c) CPC-7, d) CPC-8, e) CPC-10. Pink lines - H-bonds; green - pi-pi interactions.

In the case of SARS-CoV-2 RdRp, we had to test more protocols since basic SP/XP unguided docking 
produced poses that were correct only in part. We used the Cryo-EM structure of the SARS-CoV-2 replicating polymerase complex in the pre-catalytic state bound to inhibitor favipiravir-RTP (PDB id - 7CTT). The crystallographic pose could be reproduced using an explicit constraint on the position of the pyrazine ring with RMSD < 2.0 $\AA$ (again, the major cluster corresponded to the reference orientation), so the actual docking studies were performed using that constraint. 12 out of 16 compounds could be docked into the RdRp active site, namely CPC-1, 2, 5, 6, 7, 8, 9, 10, 13, 14, 15, and 16. Only clusters of poses resembling favipiravir-RTP. Although docking scores were lower than in the case of Mpro docking, the number and variety of interactions with the active site residue were higher. For example, CPC-14 established five $\mathrm{H}$-bonds and one pi-cation interaction with the active site residues + pi-pi interaction, H-bond and halogen interaction with RNA present in the binding pocket (Figure 7). The highest reached XP g-score was -4.47 (CPC-14); compounds CPC-16, 13, 8 , and 6 also had similar scores: $-4.17,-4.12,-4.05$, and -4.03 , respectively (XP g-score of a reference ligand was -5.23). MM-GBSA scores were also lower compared to Mpro docking: compounds CPC-6, 14, and 13 had the best $\Delta \mathrm{G}_{\text {bind }}$ values, namely $-49.8,-44.2$, and $-40.4 \mathrm{kcal} / \mathrm{mol}$, respectively. Visual inspection showed that compounds CPC-5, 6, 8, 13, 14, and 16 had the most favorable binding orientations (Figure 7). The binding pocket of RdRp has many charged residues, and compounds CPC-5, 6, 8, 13, and 14 established pi-cation interactions with R553 and R555 and, CPC-6 and CPC-7 - salt-bridges K621 and K798. Combining the interactions, docking score, and predicted $\Delta \mathrm{G}_{\text {bind }}$ values, compounds CPC-6 (Table S2), CPC-13, and CPC-14 turned to be the most promising for further developments.

a

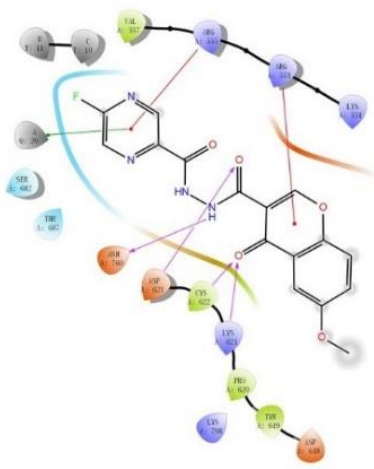

C

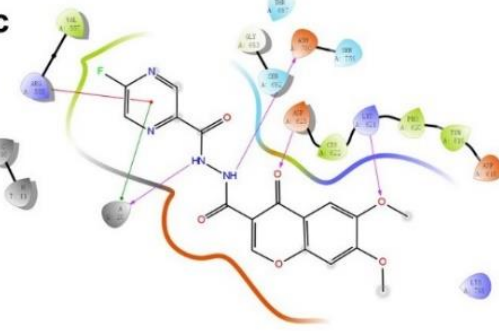

e

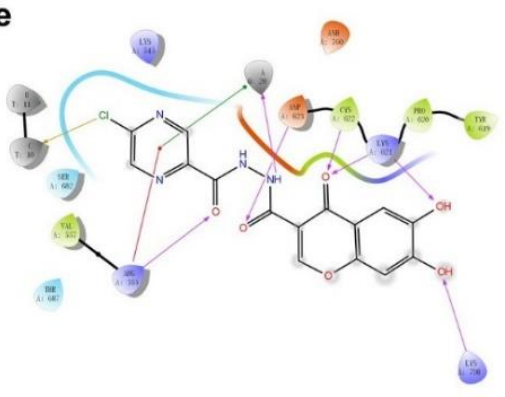

b

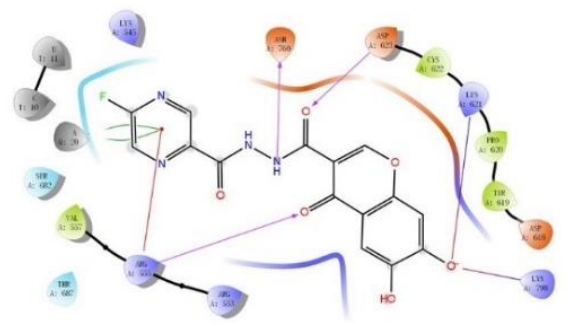

d

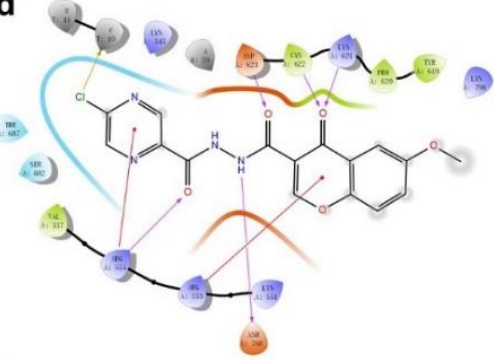

$\mathbf{f}$

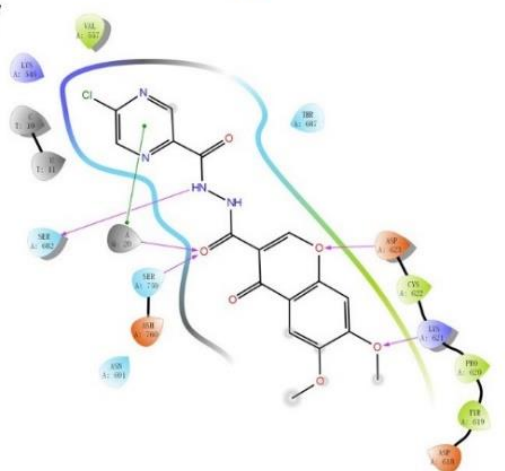

Figure 7. Interaction diagrams for the compounds with the highest predicted affinity to SARS-CoV-2 RdRp. a) CPC-5, b) CPC-6, c) CPC-8, d) CPC-13, e) CPC-14, f)CPC-16. Pink lines - H-bonds; red - pi-cation interactions; green - pi-pi interactions; pale brown - halogen interactions; red \& blue - salt bridges. 
Binding pockets of Mpro and RdRp differ significantly in their shape, volume, and physicochemical properties (Figure 8): in Mpro, the binding site is vast and shallow and has a relatively undetectable negative charge, while in RdRp, the binding pocket resembles deep and narrow tunnel packed with both negatively and positively charged residues (Figure $8 b$ ). From the dual-action side, CPC- 6 turned to be the most promising candidate. Docking studies showed that it could establish various favorable interactions with the residues in both Mpro and RdRp proteins (Figure 6, 7, 8). In the case of Mpro, CPC-6 is fixed throughout its length utilizing four H-bonds (Figure 6b), while in RdRp, CPC-6 established not only H-bonds but also salt-bridges and pication interactions with the binding pocket (Figure 7b).

a)

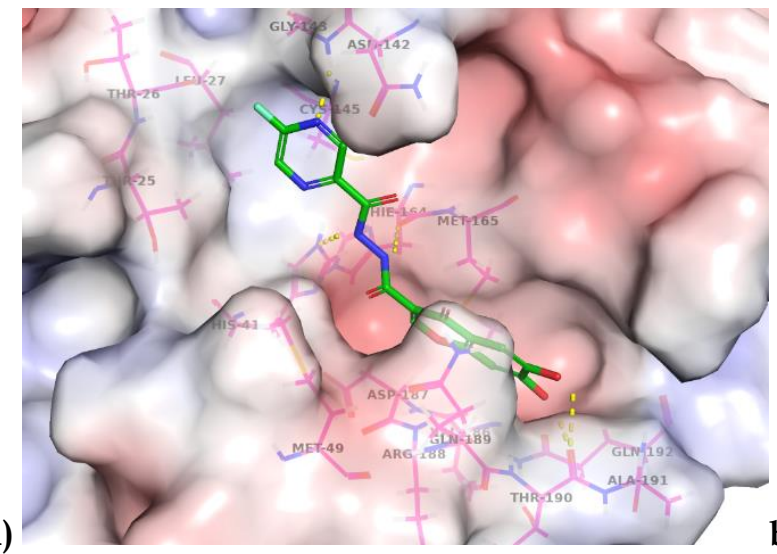

b)

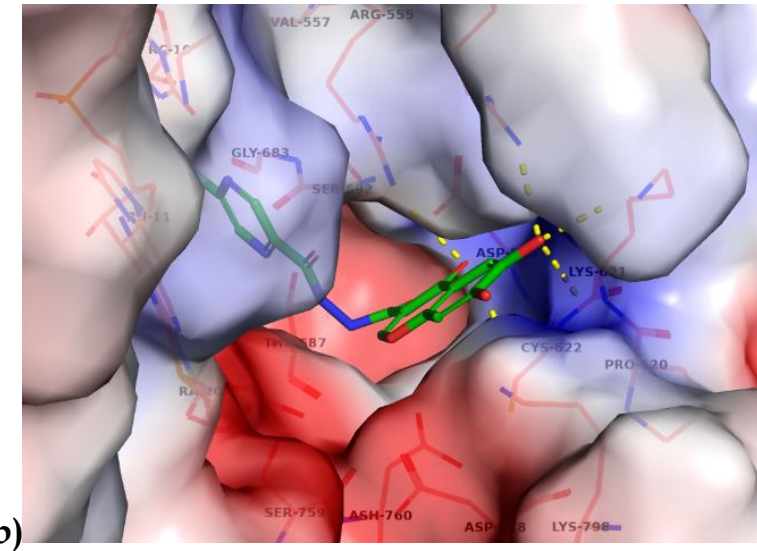

Figure 8. Binding pose of the most promising dual-acting compound CPC-6 in the active site of SARS-CoV2 Mpro (a) and RdRp (right). Yellow dashed lines indicate H-bonds, red color shows negatively charged regions, and blue corresponds to positive surface patches.

Considering the further improvement of the candidate compound CPC-6, the task seems to be challenging given the dissimilar nature of the binding pockets. However, RdRp binding tunnel still has enough unoccupied space that can be filled with additional polar groups attached to the 6,7dihydroxychromen-4-one core. To improve affinity towards the Mpro binding site, the compound can be made bulkier by adding more polar groups to occupy extra space and establish more interactions with the shallow and broad polar site. In further studies, the local chemical space around CPC-6 can be explored to identify slightly bulkier derivatives with stronger affinity to both Mpro and RdRp binding pockets.

\subsection{Synthetic accessibility \& novelty of the compounds}

The target compounds designed in this study can be synthesized according to Scheme 1 for further examination. For this purpose, firstly, CPC-1 reacts with hydrazine hydrate to obtain the corresponding hydrazide compound (CPC-2). Secondly, the 4-oxo-4H-chromene-3-carboxylic acid derivative reacts with thionyl chloride to get the corresponding compound. Finally, the target compounds can be achieved by the reaction between obtained compounds CPC-2 and CPC-4.

Since all the compounds have the same chemical scaffolds, their Tc values against a reference set of SARS-CoV-2 inhibitors were very similar. Tc values of the most novel compounds in the list, CPC-10, and CPC-11 are 0.35 each, while the least novel compound, CPC-13, has Tc equal to 0.40 . Tc value of the most promising dual-acting compound, CPC-6, is 0.37. Such low Tc values indicate that 4-oxo-N'-(pyrazine-2carbonyl)chromene-3-carbohydrazide scaffold has never been tested against SARS-CoV-2 target proteins before and, thus, represents a yet-unexplored part of chemical space. 


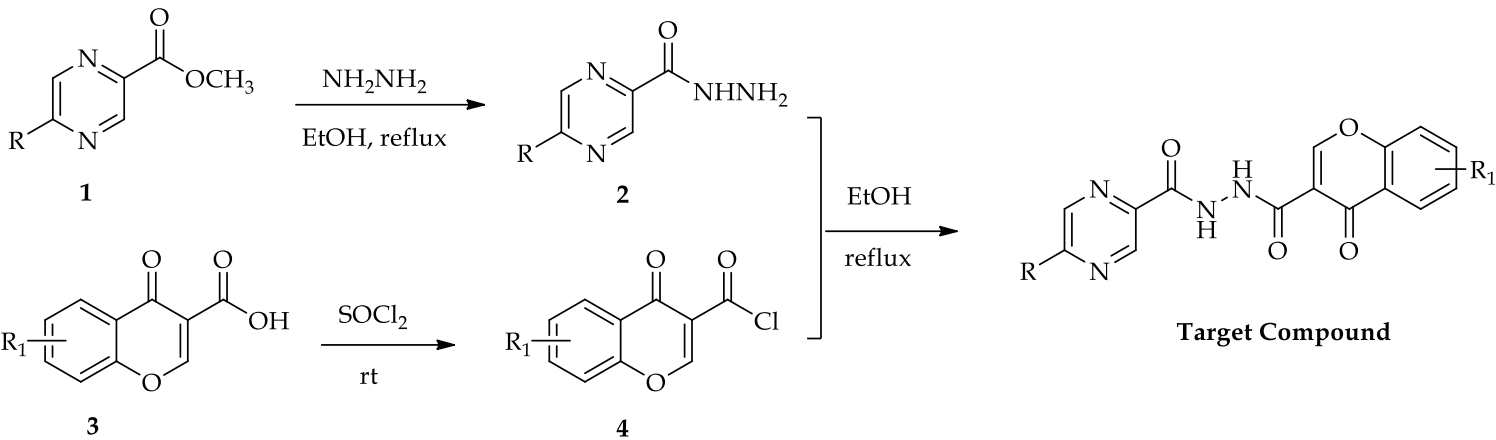

Scheme 1. Synthetic accessibility of the target compounds.

\begin{tabular}{cccccc}
\hline Compound & $\mathbf{R}$ & $\mathbf{R}_{\mathbf{1}}$ & Compound & $\mathbf{R}$ & $\mathbf{R}_{\mathbf{1}}$ \\
\hline CPC-1 & $5-\mathrm{F}$ & $6-\mathrm{OH}$ & CPC-9 & $5-\mathrm{Cl}$ & $6-\mathrm{OH}$ \\
CPC-2 & $5-\mathrm{F}$ & $6-\mathrm{CH}_{3}$ & $\mathrm{CPC}-10$ & $5-\mathrm{Cl}$ & $6-\mathrm{CH}_{3}$ \\
CPC-3 & $5-\mathrm{F}$ & $6-\mathrm{Br}$ & $\mathrm{CPC}-11$ & $5-\mathrm{Cl}$ & $6-\mathrm{Br}$ \\
CPC-4 & $5-\mathrm{F}$ & $6-\mathrm{Cl}$ & $\mathrm{CPC}-12$ & $5-\mathrm{Cl}$ & $6-\mathrm{Cl}$ \\
CPC-5 & $5-\mathrm{F}$ & $6-\mathrm{OCH}_{3}$ & $\mathrm{CPC}-13$ & $5-\mathrm{Cl}$ & $6-\mathrm{OCH}_{3}$ \\
CPC-6 & $5-\mathrm{F}$ & $6,7-\mathrm{diOH}^{2}$ & CPC-14 & $5-\mathrm{Cl}$ & $6,7-\mathrm{diOH}$ \\
CPC-7 & $5-\mathrm{F}$ & $6-\mathrm{NO}_{2}$ & CPC-15 & $5-\mathrm{Cl}$ & $6-\mathrm{NO}_{2}$ \\
CPC-8 & $5-\mathrm{F}$ & $6,7-\mathrm{diOCH}_{3}$ & CPC-16 & $5-\mathrm{Cl}$ & $6,7-\mathrm{diOCH}_{3}$ \\
\hline
\end{tabular}

\section{CONCLUSION}

This study aimed to identify new chemical entities with a dual-target activity to advance drug discovery efforts against SARS-CoV-2 infection. Using in silico approach, we created a library of hybrid compounds combining pharmacophores to the essential viral enzymes, Mpro and RdRp, and predicted that CPC-6 ( $N^{\prime}-(5-$ fluoropyrazine-2-carbonyl)-6,7-dihydroxy-4-oxo-4H-chromene-3-carbohydrazide) had good docking scores and MM-GBSA $\Delta \mathrm{G}_{\text {bind }}$ values and good interaction profiles for both Mpro and RdRp. Besides, the chemical scaffold of the compound has never been reported to inhibit the SARS-CoV-2 replication before, and it was shown to satisfy most of the drug-likeness rules, so this compound can serve as a starting point for the development of novel dual-acting drug candidates against COVID-19 infection.

\section{MATERIALS AND METHODS}

\subsection{ADME Prediction}

ADME (Adsorption, Distribution, Metabolism, and Excretion) is significant to estimate the pharmacodynamics of the designed compounds, which could be a candidate agent in drug design and discovery studies. SwissADME is a web-based platform that lets users upload or draw their hit compounds with structure or SMILES code. This tool supplies many parameters such as lipophilicity (iLOGP, XLOGP3, WLOGP, MLOGP, SILICOS-IT, Log Po/w), water solubility - Log S (ESOL, Ali, SILICOS-IT), drug-likeness rules (Lipinski, Ghose, Veber, Egan, and Muegge) and Medicinal Chemistry (PAINS, Brenk, Leadlikeness, Synthetic accessibility) methods [39]. The designed novel SARS-CoV-2 RdRp inhibitors were uploaded using SMILES codes and analyzed.

\subsection{Target Prediction}

Molecular Target investigations are crucial to determine putative phenotypical side effects or potential cross-reactivity induced by the action of a bioorganic compound of which molecular weight is not higher than $500 \mathrm{~g} / \mathrm{mol}$ [24]. SMILES codes of the designed compounds were uploaded to the Swiss Target Prediction website to analyze their putative off-targets in the human organism (https://www.swisstargetprediction.ch). 


\subsection{Toxicity Prediction}

Toxicological predictions for bioorganic compounds are essential to estimate the amount of tolerability of the hit compounds before in vitro, in vivo, and clinical studies. PKCSM is also a web-based platform for analyzing physicochemical properties of small compounds, and this online website supplies many toxicology parameters such as LD50, hERG-I inhibition, AMES toxicity, hERG-II inhibition, human maximum tolerated dose, LOAEL, skin toxicity, T. pyriformis toxicity, hepatotoxicity, and Minnow toxicity. SMILES codes of the designed compounds were uploaded to the pkCSM website to analyze their putative toxicity (http://biosig.unimelb.edu.au/pkcsm/) [32].

\subsection{Molecular Docking and Scoring}

Glide software incorporated in Schrodinger Small Molecule Suite 2020-4 was used to dock the compounds into SARS-CoV-2 Main protease (Mpro) and RNA-dependant RNA-polymerase (RdRp). Before grid generation, target protein structures (PDB ids: 6ZRT for Mpro and 7CTT for RdRp) were prepared using the Protein Preparation module in Maestro with the correction of bond orders, the addition of missing sidechains and hydrogens, and removal of crystallographic waters. Then, a restrained energy minimization under the OPLS-3 force field was applied. The active sites of the prepared proteins were defined using default parameters of receptor-grid generation (using a scaling factor of 0.8 for the Van der Waals radii of protein atoms) present in the Glide module. Grid boxes were generated around the reference ligands of 6ZRT and 7CTT complexes with the following parameters: center of grid-box - 122.38 / 124.73 / 129.18 for 7CTT and 7.24 / 16.26 / 23.41 for 6ZRT, and grid box size was adjusted to dock the ligands similar in size to the reference ligands using the corresponding option in Glide. A constrain was applied to keep the pyrazine ring in a reference position with a possible deviation $<0.6 \AA$ for the docking against RdRp. The ligands were prepared with the LigPrep module, and all possible tautomers were generated at $\mathrm{pH} 7.0 \pm 2.0$ using Epik, the specified chiralities were retained. The in-depth conformational search for each ligand was carried out with the ConfGen module using the default options. Different docking calculations were performed for each grid using a ligand Van der Waals scaling factor of 0.8 or 1.0, and sampling was performed by either SP or XP algorithms (according to control re-docking results) using the default GlideScore scoring function. MM-GBSA calculations were performed with Prime using the minimization sampling method and explicit flexibility of the 5- $\AA$ zone around the ligand. 2D interaction maps were visualized using the Ligand Interactions function in Maestro.

\subsection{Estimation of compounds novelty}

The novelty of all compounds was estimated using the Schrodinger Canvas software. We calculated the maximum pairwise Tanimoto similarity of each hit to the 7127 compounds in the ChEMBL28 database that were shown to inhibit SARS-CoV-2 (all targets) replication in vitro using the extended chemical fingerprints for four atoms (ECFP4). Those compounds with the lowest Tc values were treated as the most dissimilar (and thus novel) to already-known compounds and suitable for developing SARS-CoV-2 dual-targeting drugs.

Acknowledgments: We thank the bioinformatics (J.V. Lehtonen), translational activities, and structural biology (FINStruct) infrastructure support from Biocenter Finland and CSC IT Center for Science for computational infrastructure support at the Structural Bioinformatics Laboratory, (SBL) Åbo Akademi University. SBL is part of the NordForsk Nordic POP (Patient-Oriented Products), the Solutions for Health strategic area of Åbo Akademi University, and the InFLAMES Flagship program of the Academy of Finland on inflammation, cancer, and infection, University of Turku and Åbo Akademi University.

Author contributions: Concept - A.M.; Design - A.M., S.V.; Supervision - A.M., S.V.; Resources - A.M.; Materials A.M.; Data Collection and/or Processing - A.M., S.V.; Analysis and/or Interpretation - A.M., S.V.; Literature Search A.M., S.V.; Writing - A.M., S.V.

Conflict of interest statement: The authors declared no conflict of interest.

\section{REFERENCES}

[1] Delelis O, Carayon K, Saib A, Deprez E, Mouscadet JF. Integrase and integration: biochemical activities of HIV-1 integrase. Retrovirology. 2008; 5(5): 114-127. [CrossRef]

[2] Neamati N, Lin Z, Karki RG, Orr A, Cowansage K, Strumberg D. Metal-dependent inhibition of HIV-1 integrase. J Med Chem. 2002; 45(26): 5661-5670. [CrossRef]

[3] Kawasuji T, Yoshinaga T, Sato A, Yodo M, Fujiwara T, Kiyama R. A platform for designing HIV integrase inhibitors. 
Part 1: 2-hydroxy-3-heteroaryl acrylic acid derivatives as novel HIV integrase inhibitor and modeling of hydrophilic and hydrophobic pharmacophores. Bioorg Med Chem. 2006; 14(24): 8430-8445. [CrossRef]

[4] Johns BA, Svolto AC. Advances in two-metal chelation inhibitors of HIV integrase. Expert Opin Ther Pat. 2008; 18(11): 1225-1237. [CrossRef]

[5] Rowley M. The discovery of raltegravir, an integrase inhibitor for the treatment of HIV infection. Prog Med Chem. 2008; 46(2008): 1-28. [CrossRef]

[6] Sato M, Kawakami H, Motomura T, Aramaki H, Matsuda T, Yamashita M. Quinolone carboxylic acids as a novel monoketo acid class of human immunodeficiency virus type 1 integrase inhibitors. J Med Chem. 2009; 52(15): 48694882. [CrossRef]

[7] Elfiky AA. Ribavirin, Remdesivir, Sofosbuvir, Galidesivir, and Tenofovir against SARS-CoV-2 RNA dependent RNA polymerase (RdRp): A molecular docking study. Life Sci. 2020; 15(2020): 117592. [CrossRef]

[8] Gurung AB, Ali MA, Lee J, Farah MA, Al-Anazi KM. The potential of Paritaprevir and Emetine as inhibitors of SARSCoV-2 RdRp. Saudi J Biol Sci. 2021; 28(2): 1426-1432. [CrossRef]

[9] Katlama C, Murphy R. Dolutegravir for the treatment of HIV. Expert Opin Investig Drugs. 2008; 21(4): 523-530. [CrossRef]

[10] Di Santo R. Inhibiting the HIV integration process: past, present, and the future. J Med Chem. 2014; 57(3): 539-566. [CrossRef]

[11] Liao C, Marchand C, Burke TR, Pommier Y, Nicklaus MC. Authentic HIV-1 integrase inhibitors. Future Med Chem. 2010; 2(7): 1107-1122. [CrossRef]

[12] Sirous H, Chemi G, Gemma S, Butini S, Debyser Z, Christ F, Saghaie L, Brogi S, Fassihi A, Campiani G, Brindisi M. Identification of Novel 3-Hydroxy-pyran-4-One Derivatives as Potent HIV-1 Integrase Inhibitors Using in silico Structure-Based Combinatorial Library Design Approach. Front Chem. 2019; 7(2019): 1-20. [CrossRef]

[13] Hay M, Tomas DW, Craighead JL, Economides C, Rosenthal J. Clinical development success rates for investigational drugs. Nature Biotechnol. 2014; 32(2014): 40-51. [CrossRef]

[14] Dahlin JL, Inglese J, Walters MA. Mitigating risk in academic preclinical drug discovery. Nature Rev Drug Discov. 2015; 14(2015): 279-294. [CrossRef]

[15] Pires DEV, Blundell TL, Ascher DB. pkCSM: Predicting small-molecule pharmacokinetic and toxicity properties using graph-based signatures. J Med Chem. 2015; 58(9): 4066-4072. [CrossRef]

[16] Cheng F, Li W, Zhou Y, Shen J, Wu Z, Liu G, Lee PW, Tang Y. admetSAR: a comprehensive source and free tool for assessment of chemical ADMET properties. J Chem Inf Model. 2012; 52(11): 3099-3105. [CrossRef]

[17] Daina A, Michielin O, Zoete V. iLOGP: A Simple, robust, and efficient description of n-octanol/water partition coefcient for drug design using the GB/SA approach. J Chem Inf Model. 2014; 54(12): 3284-3301. [CrossRef]

[18] Di L, Artursson P, Avdeef A, Ecker GF, Faller B, Fischer H, Houston JB, Kansy M, Kerns EH, Krämer SD, Lennernäs $\mathrm{H}$, Sugano K. Evidence-based approach to assess passive diffusion and carrier-mediated drug transport. Drug Discov. 2012; 17(15): 905-912. [CrossRef]

[19] Zoete V, Daina A, Bovigny C, Michielin O. SwissSimilarity: A web tool for low to ultra high troughput ligand-based virtual screening. J Chem Inf Model. 2016; 56(8): 1399-1404. [CrossRef]

[20] Gfeller D. SwissTargetPrediction: a web server for target prediction of bioactive small molecules. Nucleic Acids Res. 2014; 42(W1): W32-W38. [CrossRef]

[21] Grosdidier A, Zoete V, Michielin O. SwissDock, a protein-small molecule docking web service based on EADock DSS. Nucleic Acids Res. 2011; 39(2): W270-W277. [CrossRef]

[22] Wirth M, Zoete V, Michielin O, Sauer WHB. SwissBioisostere: a database of molecular replacements for ligand design. Nucleic Acids Res. 2013; 41(D1): D1137-D1143. [CrossRef]

[23] Zoete V, Cuendet MA, Grosdidier A, Michielin O. SwissParam: a fast force feld generation tool for small organic molecules. J Comput Chem. 2011; 32(11): 2359-2368. [CrossRef]

[24] Daina A, Michielin O, Zoete V. SwissADME: A free web tool to evaluate pharmacokinetics, drug-likeness and medicinal chemistry friendliness of small molecules. Sci Rep. 2017; 7(2017): 42717-42730. [CrossRef]

[25] Liu LJ, Leung KH, Chan DSH, Wang YT, Ma DL, Leung CH. Identification of a natural product-like STAT3 dimerization inhibitor by structure-based virtual screening. Cell Death Dis. 2014; 5(6): e1293. [CrossRef] 
[26] Das S, Sarmah S, Lyndem S, Singha RA. An investigation into the identification of potential inhibitors of SARS-CoV2 main protease using molecular docking study. J Biomol Struct Dyn. 2021; 39(16): 3347-3357. [CrossRef]

[27] Kumar Y, Singh H, Natel CN. In silico prediction of potential inhibitors for the main protease of SARS-CoV-2 using molecular docking and dynamics simulation based drug-repurposing. J Infect Public Health. 2020; 13(9): 1210-1223. [CrossRef]

[28] Yu R, Chen L, Lan R, Shen R, Li P. Computational screening of antagonists against the SARS-CoV-2 (COVID-19) coronavirus by molecular docking. Int J Antimicrob Agents. 2020; 56(2): 106012. [CrossRef]

[29] Abdolmaleki A, Ghasemi JB, Ghasemi F. Computer aided drug design for multi-target drug design: SAR/QSAR, molecular docking and pharmacophore methods. Curr Drug Targets. 2017; 18(5): 556-575. [CrossRef]

[30] Mitra K, Ghanta P, Acharya S, Chakrapani G, Ramajah B, Doble M. Dual inhibitors of SARS-CoV-2 proteases: pharmacophore and molecular dynamics based drug repositioning and phytochemical leads. J Biomol Struct Dyn. 2020; 39(16); 6324-6337. [CrossRef]

[31] Keiser MJ, Roth BL, Armbruster BN, Ernsberger P, Irwin JJ, Shoichet BK. Relating protein pharmacology by ligand chemistry. Nat Biotechnol. 2007; 25(2007): 197-206. [CrossRef]

[32] Pires DE, Blundell TL, Ascher DB. PkCSM: Predicting small-molecule pharmacokinetic and toxicity properties using graphbased signatures. J Med Chem. 2015; 58(9): 4066-4072. [CrossRef]

[33] Daina A, Zoete V. A BOILED-Egg to predict gastrointestinal absorption and brain penetration of small molecules. ChemMedChem. 2016; 11(11): 1117-1121. [CrossRef]

[34] Selvam P, Chandramohan M, De Clercq E, Pannecouque C, Witrouw M. Synthesis and anti-HIV activity of 4-[(1,2dihydro-2-oxo-3H-indol-3-ylidene)amino]- $\mathrm{N}$-(4,6-dimethyl-2-pyrimidinyl)-benzene sulphonamide and its derivatives. Eur J Pharm Sci. 2001; 14(4): 313-316. [CrossRef]

[35] Sastry GM, Adzhigirey M, Day T, Annabhimoju R, Sherman W. Protein and ligand preparation: parameters, protocols, and influence on virtual screening enrichments. J Comput Aided Mol Des. 2013; 27(2013): 221-234. [CrossRef]

[36] Friesner RA, Banks JL, Murphy RB, Halgren TA, Klicic JJ, Mainz DT, Repasky MP, Knoll EH, Shelley M, Perry JK. Glide: a new approach for rapid, accurate docking and scoring. 1. Method and assessment of docking accuracy. J Med Chem. 2004; 47(7): 1739-1749. [CrossRef]

[37] Friesner RA, Murphy RB, Repasky MP, Frye LL, Greenwood JR, Halgren TA, Sanschagrin PC, Mainz DT. Extra precision glide: Docking and scoring incorporating a model of hydrophobic enclosure for protein- ligand complexes. J Med Chem. 2006; 49(21): 6177-6196. [CrossRef]

[38] Halgren TA, Murphy RB, Friesner RA, Beard HS, Frye LL, Pollard WT, Banks JL. Glide: a new approach for rapid, accurate docking and scoring. 2. Enrichment factors in database screening. J Med Chem. 2004; 47(7): 1750-1759. [CrossRef]

[39] Debnath B, Ganguly S. Synthesis, biological evaluation, in silico docking, and virtual ADME studies of 2-[2-Oxo-3(arylimino) indolin-1-yl]- $\mathrm{N}$-arylacetamides as potent anti-breast cancer agents. Monatsh Chem. 2016; 147(2016): 565574. [CrossRef] 